\title{
Managing Consumer Referrals on a Chain Network
}

\author{
Maria Arbatskaya Hideo Konishi
}

January 10, 2014

\begin{abstract}
We consider the optimal pricing and referral strategy of a monopoly that uses a simple consumer communication network (a chain) to spread product information. The first-best policy with fully discriminatory position-based referral fees involves standard monopoly pricing and referral fees that provide consumers with strictly positive referral incentives. Effective price discrimination among consumers based on their positions in the chain occurs in both the first-best solution and the second-best solution (with a common referral fee).

Keywords: communication network, consumer referral policy, referral fee, price discrimination.

JEL numbers: D4, D8, L1.

Maria Arbatskaya, Department of Economics, Emory University, Atlanta, GA 303222240. Phone: (404) 727 2770. Fax: (404) 727 4639. Email: marbats@emory.edu.

Hideo Konishi, Department of Economics, Boston College, 140 Commonwealth Avenue, Chestnut Hill, MA 02467. Tel: (617)-552-1209. Fax: (617)-552-2308. Email: hideo.konishi@bc.edu.
\end{abstract}

Acknowledgments We thank Jeong-Yoo Kim for his comments. 


\section{Introduction}

Referrals are part of our daily life. We rely on recommendations from friends and acquaintances to learn about available products and services in such diverse areas as education, medical services, construction, labor markets, financial services, telecommunications, information technology, entertainment, hospitality services, and retail trade. ${ }^{1}$ Recognizing the power of consumer referrals, ${ }^{2}$ firms try to manage the information flow in consumer communication networks by using referral policies. A consumer referral policy is a promise made by a firm to pay its current customers rewards for referring new customers. Typically, firms offer consumers rewards in the form of cash, discounts, deposits, gift certificates, bonus points, free products or services, or entry into a lottery.

In this paper, we examine how a firm should choose its price and referral fees when it relies on consumer referrals to raise consumer awareness of its product. In particular, we ask the following questions. Should the firm lower its price in an attempt to facilitate referrals? Is it optimal for the firm to offer referral payments? If yes, would the firm more than compensate consumers for making referrals, providing them with a positive expected net referral benefit? Or would the referral condition be binding for some consumers? Would the firm effectively discriminate among consumers based on their position in the referral chain? Finally, what

\footnotetext{
${ }^{1}$ For example, at Comcast, "When a friend subscribes to residential Comcast High-Speed Internet through the Refer-A-Friend program, they'll experience powerful 100\% Pure Broadband Internet and you'll enjoy one free month of residential service." At the Top of the Key basketball training company, "You are eligible for the reduced renewal rate if you have brought a friend or teammate who has also signed up for a training package" (for a Silver Package, $\$ 259$ per month is reduced to $\$ 159$ ). DIRECTV's "Spread the Word Program" offers a $\$ 100$ credit to any customer referring a friend who signs up for DIRECTV's service. The C-Loans online commercial lender guarantees "1/8th of a point on any closed commercial mortgage loan that came from your site." PeopleWare Technical Resources pays up to $\$ 500$ in referral fees for each new person placed into a contract or full-time position.

${ }^{2}$ Recommendations from personal acquaintances are by far the most relevant and trustworthy, compared with all other information sources, according to Nielsen's 2012 Global Trust in Advertising Survey of 28,000 Internet respondents from 56 countries.
} 
are the welfare implications of consumer referrals?

To answer these questions, we first consider a two-step flow of communication. ${ }^{3}$ We assume that there is one firm that can reach some consumers (called influentials or mavens) by advertising its product through mass media, and these consumers choose whether to buy the product and spread information about it to other people (called followers, imitators, or late adopters). Consumer valuations are determined stochastically. A consumer who purchases the product can at a cost refer it to one of the followers. A referral fee is paid by the firm for each referral that results in a sale.

We show that in such a model, the firm would provide influentials with a positive net referral benefit, effectively discriminating in their favor. Influentials would be better off than followers, but since the price remains unchanged after an introduction of the referral policy, referrals result in a Pareto improvement. The expected payoff of each consumer is higher because referrals increase consumer awareness, there are positive benefits to giving referrals, and the price is unchanged (compared to the standard monopoly level).

Clearly, product information can propagate for several steps in a consumer communication network. Using the framework of Jun and Kim (2008), we assume that one firm sells a product to a finite chain of $n$ consumers. A consumer receives a referral fee if the consumer's referral of the next-in-line consumer to the product results in that consumer's purchase. The monopoly firm can choose a price and the referral fees it pays for successful referrals. Jun and Kim (2008) look at the case where the firm sets a common referral fee to all consumers. In contrast, we assume that a firm could set differential referral fees for early

\footnotetext{
${ }^{3}$ The two-step model of communication was originally proposed in the 1940s to explain the effects of media on voting behavior. According to the theory, media directly affect only a small fraction of the population, and those tuned into media act as opinion leaders, influencing others' behavior.
} 
and late adopters. ${ }^{4}$

Would a firm provide higher expected referral payments to consumers who buy earlier? We find that this is the case. When the firm can charge different referral fees based on consumers' positions (the first-best problem), both the probability of purchase and the expected referral payment are decreasing along the referral chain (Theorem 1). The result is intuitive. The last consumer (consumer $n$ ) cannot make a referral, and her purchase of the product and referral do not generate extra sales. The second-to-last consumer's purchase has an externality since her purchase may lead to consumer $n$ 's purchase, but the externality is limited only to sales made to her successor. For consumers positioned earlier in the chain, the externality is larger. That is, early buyers are more valuable to the firm than later buyers since their purchase of the product is necessary for the referral chain to continue, and the potential gains from a longer chain are larger.

This intuition is the same as in the second-best problem of Jun and Kim (2008), in which the firm has to set a common price $p$ and referral fee $r$ to all consumers. Since in their framework $n$ purchase probabilities (variables $\alpha_{1}, \ldots, \alpha_{n}$ ) need to be controlled by two policy tools $p$ and $r$, finding an optimal choice of $(p, r)$ can be a highly nonconvex problem with multiple local maxima. This means that although intuitively it may be beneficial for the firm to effectively discriminate between consumers based on their positions in a chain, the stationary outcome is another plausible candidate for the optimal solution, especially for large $n$. It is easy to show that purchase probabilities must be equal (which also implies that there is no discrimination among consumers) if the referral condition is binding even

\footnotetext{
${ }^{4}$ After we completed the current paper, Jeong-Yoo Kim let us know about his unpublished note that considers the first-best problem for the special case of $n=3$.
} 
for one consumer. With their unusual tie-breaking rule, Jun and Kim (2008) assume away the stationary outcome. ${ }^{5}$ We revisit the second-best problem of Jun and Kim in Appendix $\mathrm{B}$, where we prove that such a stationary outcome is not optimal.

In sum, we show that both in the first-best and the second-best scenarios, the firm would choose to discriminate between consumers based on their positions in the communication chain and would provide positive expected net referral benefits to all consumers in a position to refer. Despite the simplicity of the model, the proofs are rather involved. Techniques developed in this paper may be useful for other purposes.

The next section is devoted to the simplest first-best problem: the influentials-andfollowers model. In Section 2, we analyze the first-best problem for a chain of any finite length. We discuss the results in Section 3 and conclude in Section 4. Appendix A contains proofs for all the statements for the first-best problem. In Appendix B, we present results for the second-best problem of Jun and Kim (2008), in which the firm charges a common referral fee to all consumers.

\section{Influentials-and-Followers Model $(n=2)$}

In this section, we consider a two-step model of communication, in which influentials (informed by the firm through advertising) can buy the product and recommend it to other potential consumers. While the problem is interesting in its own right, it also provides some intuition for the general case of consumer chain networks of length $n$, presented in the next

\footnotetext{
${ }^{5}$ Jun and Kim (2008) say nothing about the case where the referral condition is binding: $r(1-F(p))=\rho$ ( or $r \alpha_{n}=\rho$ ). They assume that if a consumer is indifferent between making and not making a referral, then she will not make a referral. This tie-breaking rule is convenient since it directly implies that consumers make referrals if and only if there are positive incentives for referral (their Proposition 2). Thus, a stationary outcome is ruled out as it is not compatible with active referrals. Here, we are assuming a tie-breaking rule that allows for referrals to be given in the stationary outcome. That is, we assume that if a consumer is indifferent between making and not making a referral, she refers.
} 
section. As we restrict the number of referrals to one, ${ }^{6}$ we can normalize the number of influentials and followers to one and refer to them as consumer 1 and consumer 2 .

The firm chooses a price $p \geq 0$ and referral fee $r \geq 0$ to maximize its profits from selling a product it produces at a marginal $\operatorname{cost} c \geq 0$. Each consumer's willingness-to-pay $v$ is an i.i.d. random variable drawn from a twice continuously differentiable distribution function $F(v)$ over $[\underline{v}, \bar{v}]$ with density function $f(v)$. Denote by $\alpha_{k}$ the probability that consumer $k$ buys the product conditional on being introduced to it, $k=1,2$. Consumers need to pay a $\operatorname{cost} \rho>0$ to make one referral, which yields a referral fee $r$ if it results in a sale. Consumers make their purchase and referral decisions to maximize their expected utility. We assume that when indifferent between making and not making a referral, consumers refer. That is, consumer 1 refers whenever $\alpha_{2} r \geq \rho$.

Let $D(p)=1-F(p)$ and $P(\alpha)=D^{-1}(\alpha)$ be the standard demand and inverse demand functions. We assume that the profit function without referrals, $\pi(\alpha) \equiv \alpha(P(\alpha)-c)$, is concave. Let $\alpha^{m}$ be the standard monopoly output, $\alpha^{m}=\arg \max \{\alpha(P(\alpha)-c)\}$, and let $p^{m}=P\left(\alpha^{m}\right)$ and $\pi^{m}=\alpha^{m}\left(p^{m}-c\right)$ be the associated monopoly price and profit. We will assume that $\pi^{m}>\rho$, which (according to Proposition 1 below) guarantees that the firm supports referrals. The purchase probabilities are then $\alpha_{1}=D\left(p-\alpha_{2} r+\rho\right) \geq 0$ and $\alpha_{2}=D(p) \geq 0$

The firm chooses a strategy $(p, r)$ to maximize its profits

$$
\begin{aligned}
\hat{\Pi}(p, r) & =(p-c) \alpha_{1}+(p-r-c) \alpha_{1} \alpha_{2} \\
& =\left(p-c-r \alpha_{2}\right) \alpha_{1}+(p-c) \alpha_{1} \alpha_{2} .
\end{aligned}
$$

\footnotetext{
${ }^{6}$ The analysis and results are similar when this assumption is relaxed. See Section 4 for the discussion.
} 
From the expressions for $\alpha_{1}$ and $\alpha_{2}$,

$$
p=P\left(\alpha_{2}\right)
$$

and

$$
r=\frac{P\left(\alpha_{2}\right)-P\left(\alpha_{1}\right)+\rho}{\alpha_{2}} .
$$

We can write the profits as a function of only $\alpha_{1}$ and $\alpha_{2}$ :

$$
\Pi\left(\alpha_{1}, \alpha_{2}\right)=\left(P\left(\alpha_{1}\right)-c-\rho\right) \alpha_{1}+\left(P\left(\alpha_{2}\right)-c\right) \alpha_{1} \alpha_{2} .
$$

The following proposition describes the optimal (profit-maximizing) solution for monopoly.

Proposition 1. The firm supports consumer referrals whenever $\pi^{m}>\rho$. It sets the standard monopoly price and provides influentials with a positive net referral benefit, effectively discriminating in their favor. Consumer referrals result in a Pareto improvement. The purchase probability of influentials is higher than that of followers, and both probabilities decrease in referral and production costs. Assuming that demand is not too convex, the firm sets a higher referral fee and supports higher expected referral payments when referral cost is higher. When consumer valuations are uniformly distributed over $[0,1]$, the optimal referral fee and expected referral payment decrease in production cost.

Proposition 1 states that the presence of consumer referrals does not affect the price in this model. The price is the standard monopoly price $p^{m}$, which increases with the marginal cost $c$ and is independent of the referral cost $\rho$. Both influentials and followers are better off when referrals exist because of an increase in consumer awareness about the product, 
positive net benefits to giving referrals, and no price change. Assuming that demand is not too convex, ${ }^{7}$ the firm compensates influentials for the higher cost of referral with a higher referral fee and expected referral benefit. At least in the case of the uniform distribution of valuations, the referral fee decreases in production cost.

\section{The First-Best Problem for a Finite Chain Network}

We maintain the modeling assumptions of the two-step communication model, extending it to the case of a communication chain of length $n \geq 2$. Suppose a firm can set referral fees conditional on consumer location in the communication network. That is, the policy tools are $p$ and $r_{2}, \ldots, r_{n}$, where $r_{k}$ is the referral fee that consumer $k-1$ receives if consumer $k$ purchases the product following her referral. Consumer $k$ 's probability of purchase when she is informed about the product is determined by the firm's policy as follows: $\alpha_{n}=D(p)=1-F(p) \geq 0$ and $\alpha_{k}=D\left(p-\alpha_{k+1} r+\rho\right) \geq 0$ for $k=1, \ldots, n-1$.

The monopoly profit with active consumer referrals is

$$
\widehat{\Pi}=\sum_{k=2}^{n}\left[\left(p-c-r_{k} \alpha_{k}\right)\left(\prod_{\ell=1}^{k-1} \alpha_{\ell}\right)\right]+(p-c) \prod_{\ell=1}^{n} \alpha_{\ell} .
$$

From the expressions for purchase probabilities, we find that

$$
p=P\left(\alpha_{n}\right)
$$

and

$$
r_{k}=\frac{P\left(\alpha_{n}\right)-P\left(\alpha_{k-1}\right)+\rho}{\alpha_{k}}
$$

for $k=2, \ldots, n$, where $P(\alpha)=D^{-1}(\alpha)$.

\footnotetext{
${ }^{7}$ Although we analyze a monopoly problem, the condition is exactly the same as the condition for strategic substitutes in the Cournot oligopoly problem.
} 
We can then describe the problem in terms of $\alpha_{k}$ s only. The firm's profit can be written

as

$$
\Pi=\sum_{k=1}^{n-1}\left[\left(P\left(\alpha_{k}\right)-c-\rho\right)\left(\prod_{\ell=1}^{k} \alpha_{\ell}\right)\right]+\left(P\left(\alpha_{n}\right)-c\right) \prod_{\ell=1}^{n} \alpha_{\ell} .
$$

Let $\alpha_{k}^{*}$ denote the profit-maximizing probability of buying for consumer $k, k=1, \ldots, n$. It is easy to see what the optimal probability of buying is for consumer $n$. Taking the first-order condition with respect to $\alpha_{n}$, we obtain

$$
\frac{\partial \Pi}{\partial \alpha}=(M R(\alpha)-c)\left(\prod_{\ell=1}^{n-1} \alpha_{\ell}\right)=0
$$

where $M R(\alpha) \equiv P(\alpha)-\alpha P^{\prime}(\alpha)$ is the marginal revenue.

This implies that for any $n, \alpha_{n}^{*}=\alpha^{m}$ and the optimal monopoly price is $p^{*}=p^{m}$ as long as $\alpha_{k}>0$ for $k=1, \ldots, n-1$. This observation is quite sensible: no matter how many consumers there are, the last consumer does not make a referral, and the firm should charge the monopoly price for her. The main results for the optimal monopoly policy are stated in Proposition 2.

Proposition 2. For any n, the firm's optimal policy supports consumer referrals whenever $\pi^{m}>\rho$, and it is such that $\alpha_{1}^{*}>\alpha_{2}^{*}>\ldots>\alpha_{n}^{*}=\alpha^{m}$. The firm sets the standard monopoly price $p^{*}=p^{m}$ and provides all referring consumers with a positive net referral benefit such that $\alpha_{2}^{*} r_{2}^{*}>\alpha_{3}^{*} r_{3}^{*}>\ldots>\alpha_{n}^{*} r_{n}^{*}>\rho$, effectively discriminating in favor of consumers located earlier in the chain. Consumer referrals result in a Pareto improvement.

The formal proof of Proposition 2 is in Appendix A, but we provide a sketch of the proof here. To prove Proposition 2, we analyze the problem where $k$ consumers are left in the chain and solve it recursively by backward induction. Let $V(k)$ be the optimal profit from 
the last $k$ consumers, and let $\gamma^{*}(k)$ be the profit-maximizing purchase probability of the $k$ th to last consumer. Since $\gamma^{*}(1)=\alpha^{m}$, the expected profit from the last consumer reached by referral is the simple monopoly profit, $V(1)=\pi^{m}$.

The optimal solutions $V(k)$ and $\gamma^{*}(k)$ when $k$ consumers are left in the chain can be defined recursively:

$$
V(k)=\max _{\alpha}\{\alpha[P(\alpha)-c-\rho+V(k-1)]\}
$$

and

$$
\gamma^{*}(k)=\arg \max _{\alpha}\{\alpha[P(\alpha)-c-\rho+V(k-1)]\}
$$

for $k \geq 2$. Lemma 1 shows that the optimal purchase probability for the $k$ th to last consumer $\gamma^{*}(k)$ is an increasing sequence of $k: \gamma^{*}(k+1)>\gamma^{*}(k)$ for all $k$, assuming the optimal profit is increasing with the number of consumers left, i.e. $V(k+1)>V(k)$ for all $k$.

Lemma 1. Suppose that $\pi^{m}>\rho$ and $V(k+1)>V(k)$ for any $k \geq 1$. Then, $\gamma^{*}(k+1)>$ $\gamma^{*}(k)$ holds for all $k \geq 1$.

Under the assumption of an increasing profit sequence, we can use Lemma 1 to characterize the optimal purchase probability sequence: $\alpha_{k}^{*}=\gamma^{*}(n-k+1)$ for any fixed $n \geq 2$ and all $k=1, \ldots, n$ because the $k$ th consumer from the top is the $(n-k+1)$ th consumer from the bottom: i.e., $\alpha_{1}^{*}>\alpha_{2}^{*}>\ldots>\alpha_{n}^{*}$.

Corollary 1. Suppose that $\pi^{m}>\rho$ and $V(k+1)>V(k)$ for any $k \geq 1$. Then, for all $n$, under the optimal strategy, the probability of purchase declines along the referral chain: i.e., $\alpha_{1}^{*}>\alpha_{2}^{*}>\ldots>\alpha_{n}^{*}$ 
Lemma 2 shows that the optimal profit sequence $V(k)$ is indeed increasing as long as $\pi^{m}>\rho$.

Lemma 2. Suppose that $\pi^{m}>\rho$. Monopoly profit increases in the length of the consumer chain: $V(k)<V(k+1)$ for all $k \geq 1$.

Lemma 2 is proved by induction in Appendix A. There we suppose that $V(k)>\ldots>V(1)$. Then, looking at the monopoly problem with $k+1$ consumers, we show that the firm can achieve higher profits $V(k+1)>V(k)$ if it applies the optimal policy for $k$ to the first $k$ consumers and provides consumer $k$ with just enough incentives to make a referral to consumer $k+1$ (which is profitable because $\pi^{m}>\rho$ and consumer $k+1$ will face the monopoly price). This proves that $V(k)$ is an increasing sequence. Putting together Corollary 1 and Lemma 2, we conclude that $\alpha_{1}^{*}>\alpha_{2}^{*}>\ldots>\alpha_{n}^{*}=\alpha^{m}$, and from (7) we have $\alpha_{2}^{*} r_{2}^{*}>\alpha_{3}^{*} r_{3}^{*}>$ $\ldots>\alpha_{n}^{*} r_{n}^{*}>\rho$. The next proposition presents some comparative statics results.

Proposition 3. Suppose that $\pi^{m}>\rho$. The purchase probabilities and profits decrease in referral and production costs. The price decreases in production cost but is independent of referral cost. The net referral benefits and consumer payoffs decrease in referral cost.

As expected, the firm sells less and has lower profits when costs increase. The firm favors lower referral and production costs. The optimal price is the standard monopoly price. It is insensitive to referral cost and decreases with production cost. Consumers face higher (expected) net referral benefits and consumer payoffs when the cost of referral goes down. If we assume that the distribution of values is uniform, we can derive further results. We can show that not only is the expected referral benefit decreasing in consumer location in the 
chain (Proposition 2), but the referral fees themselves are lower for later buyers: $r_{k+1}^{*}<r_{k}^{*}$ for $k=2, \ldots, n-1$.

Proposition 4. Suppose that $\pi^{m}>\rho$ and consumer valuations are uniformly distributed over $[0,1]$. Then the optimal referral fees satisfy $r_{2}^{*}>r_{3}^{*}>\ldots>r_{n}^{*}>0$. Both referral fees and expected referral payments decrease in production costs.

Figure 1 illustrates the solution for a chain of length $n=5$, the uniform distribution of values, $\rho=0.01$, and $c=0$. Figure 1 plots the optimal profits starting at the $k$ th consumer (when there are $n-k+1$ consumers left in the chain), $V(n-k+1)$. As expected, the profits increase with the number of consumers left in the chain, $V(1)<V(2)<\ldots<V(5)$. Figure 1 also shows that the purchase probability $\alpha_{k}^{*}$, referral fee $r_{k}^{*}$, and the expected referral payments $\alpha_{k}^{*} r_{k}^{*}$ for the $k$ th consumer are all decreasing in $k$. Comparative statics results for the referral cost $\rho$ and production cost $c$ are presented in Figures 2 and 3. Panels $\mathrm{A}, \mathrm{B}$, and $\mathrm{C}$ of each figure depict purchase probabilities, referral fees, and referral payments, respectively. From Panel $\mathrm{C}$ of Figure 2, it is clear that the expected referral payments may decrease with referral cost for consumers positioned early in the chain. The reason is that although referral fees are shown to increase with referral cost (Panel B of Figure 2), the purchase probabilities always decrease in referral cost (Proposition 3). The overall impact of referral cost on the expected referral payments depends on the consumer's location in the communication network. 


\section{Conclusion}

With the advancement of Internet technology, the data on consumer positions in social networks become more easily available. Firms can now identify influential persons within a network with greater precision. Indeed, Google and Microsoft, among other companies, have obtained patents on systems for identifying nodes in social networks for more targeted ad delivery. ${ }^{8}$ Firms should then utilize this new capability of targeting specific consumers in social networks to effectively discriminate between consumers based on their position in the network. Our paper suggests how referral fees should be set in this case. Importantly, we find that the firm discriminates in favor of more influential customers. This is consistent, for example, with the practice by Amazon to offer free products to its top reviewers (Amazon Vine members).

The optimal price-referral fee mix depends on whether the number of firm's tools is sufficient to achieve the first-best. This is the case in the two-step model of communication, in which opinion leaders serve as intermediaries between the mass media and other potential consumers. This is also the case when referral fees can vary with consumer position on the referral chain. In these cases, we find that the standard monopoly price is preserved. The result is important because it implies that there is no downside to consumer referrals. If a firm supports consumer referrals, it always benefits consumers as well.

In the second-best problem by Jun and Kim (2008), they assume that the price of the product is non-discriminatory, and the referral fee is constant no matter where a consumer is located on the chain. In contrast with our first-best solution, only partial price discrimination

\footnotetext{
${ }^{8}$ Microsoft's patent "Identifying influential persons in a social network" was filed in September 2006 and published March 2008. Google's patent "Network node ad targeting" was filed by Google in December 2006 and published in July 2008.
} 
can be achieved with a limited number of tools. In Appendix B, we revisit the second-best problem of Jun and Kim (2008). As in the case of the first-best problem, two qualitatively different referral equilibria could possibly arise. The one described by Jun and Kim (the nonstationary outcome) is characterized by a nonbinding referral condition, unequal probabilities of purchase, and price discrimination among consumers. The other one (the stationary outcome) involves a binding referral condition, equal probabilities of purchase, and no price discrimination among consumers. We strengthen Jun and Kim's findings by showing that even if we allow for the stationary outcome to arise, it cannot be optimal, even locally. The second-best solution is therefore qualitatively similar to the first-best solution in that the firm effectively discriminates between consumers based on their position in the chain.

In the first-best model, intuitive comparative statics results arise. Since the price is set at the standard monopoly level, it is increasing in the marginal cost and independent of the referral cost and chain length. This is in contrast with the results obtained for the second-best model by Jun and Kim (2008). Using numerical simulations, they show that the optimal product price is non-monotonic in chain length and referral and production costs. We also find that higher production costs prompt the firm to reduce buying probabilities and, at least for the uniform distribution of values, referral fees as well. A consumer who has a longer chain of followers buys more often. Such a consumer obtains higher expected net referral benefit and payoff.

Although we restrict our attention in this paper to a simple chain network, the methods developed here can be applied to more general communication networks. For example, we can extend the model by allowing consumers to make multiple referrals, with heterogeneous quotas for different consumers. As long as the consumer network is a finite tree, our main 
first-best results continue to hold. As in this paper, backward induction would need to be used to derive the optimal policy for the firm.

If we allow the firm to make a decision about the intensity of advertising, it is clear that the firm will advertise more when the profit from the consumer referral chain is larger. The firm's profit would be higher when the cost of production and referral fee are lower and the consumer chain is longer. Hence, if it becomes cheaper to make referrals (perhaps due to the advent of a new social media technology), firms would advertise more and reach more consumers through referrals. In the model, mass advertising is not displaced by more efficient consumer referrals. Instead, traditional advertising is more beneficial when profits from consumer referral chains increase due to a lower referral cost. ${ }^{9}$

It would be interesting to combine the above observations with targeted advertising. Among other applications, Galeotti and Goyal (2009) consider a problem of targeted advertising by monopoly on a general consumer communication network. They analyze the firm's problem of choosing the intensity of advertising based on the number of people a consumer obtains information from and spreads information to. For simplicity, they assume away the firm's pricing and consumer referral decisions and do not allow referral chains. In their model, every informed consumer purchases the product and refers to all the contacts under her influence. They show that in its advertising strategy, the firm should target the marginalized consumers (those who seek information from few people) and influentials (consumers who inform many people). This is a very interesting result. It is intuitive because the

\footnotetext{
${ }^{9}$ Suppose that by spending $C(x)$, the firm can reach a fraction $x$ of consumers through advertising. Then, the firm's profits are $\widetilde{\Pi}=x V(n)-C(x)$, where $V(n)$ is the optimal profit for a consumer chain of size $n$, given referral and production costs $\rho$ and $c$. Since $V(n)$ is increasing in $n$ and decreasing in $\rho$ and $c$, it follows immediately that the firm would choose to advertise more when the consumer chain size $n$ is high and referral and production costs $\rho$ and $c$ are low.
} 
marginalized consumers are not likely to be informed by word-of-mouth, while influentials have a higher network value in terms of the future sales they generate.

In contrast to Galeotti and Goyal (2009), we look at a chain network and allow for chain referrals. Still, we can say something about targeted advertising in the context of our chain model. Suppose the firm can set a price and also choose an advertising reach to each consumer. Following Galeotti and Goyal, assume that consumers who purchase the product always refer. We can show that, although the firm would choose to advertise to all consumers, the firm should advertise more to consumers located earlier in the chain.

In the spirit of the models with opinion leaders and followers, in this paper we did not consider the possibility of the same person being reached through both advertising and referrals. When consumers can receive multiple advertisements and/or referrals, the issue of congestion has to be carefully addressed. Anderson and de Palma (2009), among others, study congestion in advertising messages, and Arbatskaya and Konishi (2013) consider congestion in consumer referrals. It would be also interesting to extend the model by allowing consumer referrals to be targeted. Since consumers would choose to refer their most promising contacts, this would make referrals more efficient and in turn increase the benefit of advertising. We leave such analyses for future work. 


\section{Appendix A}

Proof of Proposition 1. Assuming that the firm supports consumer referrals, it follows from (4) that for any $\alpha_{1}>0$ the firm sets

$$
\alpha_{2}^{*}=\underset{\alpha}{\arg \max }\{\pi(\alpha)\}
$$

where $\pi(\alpha)=(P(\alpha)-c) \alpha$, and the first-order condition for $\alpha_{2}$ implies $\pi^{\prime}\left(\alpha_{2}\right)=0$. That is, the firm sets the standard monopoly output and price: $\alpha_{2}^{*}=\alpha^{m}$ and $p^{*}=p^{m}$.

At the optimal $\alpha_{2}^{*}=\alpha^{m}$, the profits are

$$
\Pi\left(\alpha_{1}, \alpha^{m}\right)=\left(P\left(\alpha_{1}\right)-c-\rho+\pi^{m}\right) \alpha_{1} .
$$

The first-order condition for $\alpha_{1}$ is then:

$$
\frac{\partial \Pi\left(\alpha_{1}, \alpha^{m}\right)}{\partial \alpha_{1}}=\pi^{\prime}\left(\alpha_{1}\right)+\pi^{m}-\rho=0 .
$$

Hence, as long as $\pi^{m}>\rho$, influentials buy more often than followers: $\alpha_{1}^{*}>\alpha_{2}^{*}$. Note that this implies that influentials obtain a positive net referral benefit $\alpha_{2}^{*} r-\rho=P\left(\alpha_{2}^{*}\right)-P\left(\alpha_{1}^{*}\right)>0$, and are overall better off than followers (and than themselves in the case of no referrals).

From (12) and (13), we find that $\frac{\partial \alpha_{2}^{*}}{\partial c}=\frac{1}{\pi^{\prime \prime}\left(\alpha_{2}^{*}\right)}<0, \frac{\partial \alpha_{2}^{*}}{\partial \rho}=0, \frac{\partial \alpha_{1}^{*}}{\partial c}=-\frac{\frac{d^{2} \Pi\left(\alpha_{1}^{*}, \alpha_{2}^{*}\right)}{d c d \alpha_{1}^{*}}}{\pi^{\prime \prime}\left(\alpha_{1}^{*}\right)}=\frac{1+\alpha_{2}^{*}}{\pi^{\prime \prime}\left(\alpha_{1}^{*}\right)}<$ 0 , and $\frac{\partial \alpha_{1}^{*}}{\partial \rho}=-\frac{\frac{d^{2} \Pi\left(\alpha_{1}^{*}, \alpha_{2}^{*}\right)}{d \rho d \alpha_{1}}}{\pi^{\prime \prime}\left(\alpha_{1}^{*}\right)}=\frac{1}{\pi^{\prime \prime}\left(\alpha_{1}^{*}\right)}<0$. To determine how the expected referral payments and referral fee depend on production and referral costs, we use (3) to obtain

$$
\frac{d\left(\alpha_{2}^{*} r^{*}\right)}{d \rho}=\frac{d\left(P\left(\alpha_{2}^{*}\right)-P\left(\alpha_{1}^{*}\right)+\rho\right)}{d \rho}=1-P^{\prime}\left(\alpha_{1}^{*}\right) \frac{\partial \alpha_{1}^{*}}{\partial \rho}=1-\frac{P^{\prime}\left(\alpha_{1}^{*}\right)}{\pi^{\prime \prime}\left(\alpha_{1}^{*}\right)}
$$

From $\pi^{\prime \prime}(\alpha)=\alpha P^{\prime \prime}(\alpha)+2 P^{\prime}(\alpha)$, it follows that $\frac{d\left(\alpha_{2}^{*} r^{*}\right)}{d \rho}=\alpha_{2}^{*} \frac{d r^{*}}{d \rho}>0$ whenever $\alpha_{1}^{*} P^{\prime \prime}\left(\alpha_{1}^{*}\right)+$ $P^{\prime}\left(\alpha_{1}^{*}\right)<0$. Assuming this condition on demand holds, as in the case of the uniform distribution of values, we find that $\frac{d r^{*}}{d \rho}>0$. 
Similarly, we can derive

$$
\begin{aligned}
\frac{d\left(\alpha_{2}^{*} r^{*}\right)}{d c} & =\frac{d\left(P\left(\alpha_{2}^{*}\right)-P\left(\alpha_{1}^{*}\right)+\rho\right)}{d c} \\
& =P^{\prime}\left(\alpha_{2}^{*}\right) \frac{\partial \alpha_{2}^{*}}{\partial c}-P^{\prime}\left(\alpha_{1}^{*}\right) \frac{\partial \alpha_{1}^{*}}{\partial c} \\
& =\frac{P^{\prime}\left(\alpha_{2}^{*}\right)}{\pi^{\prime \prime}\left(\alpha_{2}^{*}\right)}-\frac{P^{\prime}\left(\alpha_{1}^{*}\right)}{\pi^{\prime \prime}\left(\alpha_{1}^{*}\right)}\left(1+\alpha_{2}^{*}\right)
\end{aligned}
$$

and

$$
\alpha_{2}^{*} \frac{d r^{*}}{d c}=\frac{P^{\prime}\left(\alpha_{2}^{*}\right)}{\pi^{\prime \prime}\left(\alpha_{2}^{*}\right)}-\frac{P^{\prime}\left(\alpha_{1}^{*}\right)}{\pi^{\prime \prime}\left(\alpha_{1}^{*}\right)}\left(1+\alpha_{2}^{*}\right)-\frac{r}{\pi^{\prime \prime}\left(\alpha_{2}^{*}\right)} .
$$

For the uniform $U[0,1]$ distribution of values, $\pi\left(\alpha_{2}\right)=\left(1-\alpha_{2}-c\right) \alpha_{2}, \alpha_{2}^{*}=\frac{1-c}{2}, p^{*}=$ $\frac{1+c}{2}, \pi^{m}=\left(\frac{1-c}{2}\right)^{2}, \alpha_{1}^{*}=\frac{1}{2}\left(1-c-\rho+\pi^{m}\right), r^{*}=\frac{\rho+\pi^{m}}{1-c}=\frac{\rho}{1-c}+\left(\frac{1-c}{4}\right)$, and $\alpha_{2}^{*} r^{*}-\rho=$ $\alpha_{1}^{*}-\alpha_{2}^{*}=\frac{1}{2}\left(\pi^{m}-\rho\right)$, where $\pi^{m}=\frac{1}{4}(1-c)^{2}$. The firm would support consumer referrals if $\Pi\left(\alpha_{1}^{*}, \alpha_{2}^{*}\right)>\pi^{m}$, which holds whenever $\pi^{m}>\rho$. Since $\pi^{\prime \prime}(\alpha)=-2, P^{\prime}(\alpha)=-1$, we find that $\frac{d\left(\alpha_{2}^{*} r^{*}\right)}{d \rho}=\alpha_{2}^{*} \frac{d r^{*}}{d \rho}=\frac{1}{2}>0, \frac{d\left(\alpha_{2}^{*} r^{*}\right)}{d c}=-\frac{1}{2} \alpha_{2}^{*}<0$, and $\frac{d r^{*}}{d c}=\frac{\rho}{(1-c)^{2}}-\left(\frac{1}{4}\right)<0$ because $\pi^{m}=\frac{(1-c)^{2}}{4}>\rho$.

Proof of Lemma 1. We take four steps to prove Lemma 1.

1. Consider a chain of length $n=1$. The profit-maximizing probability $\gamma^{*}(1)=\alpha^{m}$ satisfies $M R\left(\gamma^{*}(1)\right)=c$, where $M R(\alpha)=(\alpha P(\alpha))^{\prime}=P(\alpha)+\alpha P^{\prime}(\alpha)$ is the marginal revenue from extending sales.

2. Next consider $n=2$. In this case, the optimization problem is

$$
V(2)=\max _{\alpha}[\alpha(P(\alpha)-c-\rho)+\alpha V(1)]
$$

and $V(2)>V(1)$ implies that there is an optimal solution $\gamma^{*}(2)$ in this problem. Since $M R(\alpha)$ is decreasing, the following first-order condition characterizes $\gamma^{*}(2)$ :

$$
M R\left(\gamma^{*}(2)\right)-c-\rho+V(1)=0
$$


Since $V(1)=\pi^{m}>\rho, M R(\alpha)$ is decreasing, and $M R\left(\gamma^{*}(1)\right)-c=0$, we conclude that $\gamma^{*}(2)>\gamma^{*}(1)$

3. Suppose that $\gamma^{*}(1)<\ldots<\gamma^{*}(k-1)$ for $k \geq 3$. We will show that $\gamma^{*}(k-1)<\gamma^{*}(k)$ holds. By definition, we have

$$
V(k)=\max _{\alpha}[\alpha(P(\alpha)-c-\rho)+\alpha V(k-1)]
$$

and the first-order condition for $\gamma^{*}(k)$ is

$$
M R\left(\gamma^{*}(k)\right)-c+V(k-1)-\rho=0
$$

The first-order condition for $\gamma^{*}(k-1)$ is

$$
M R\left(\gamma^{*}(k-1)\right)-c+V(k-2)-\rho=0
$$

Since $M R(\alpha)$ is decreasing and $V(k-2)<V(k-1)$, we conclude that $\gamma^{*}(k-1)<\gamma^{*}(k)$ holds.

4. By an induction argument, we complete the proof. $\square$

Proof of Corollary 1. If $n$ consumers are left in the chain, then the probability of purchase for the first consumer is $\alpha_{1}^{*}=\gamma^{*}(n)$. If the second consumer is successfully referred to the product, then $n-1$ consumers are left in the chain, and the probability of purchase for the second consumer is $\alpha_{2}^{*}=\gamma^{*}(n-1)$. Similarly, $\alpha_{k}^{*}=\gamma^{*}(n-k+1)$ for all $k=1, \ldots, n$. The probability of purchase declines along the referral chain (i.e. $\alpha_{1}^{*}>\alpha_{2}^{*}>\ldots>\alpha_{n}^{*}$ ) because $\gamma^{*}(n)>\gamma^{*}(n-1)>\ldots>\gamma^{*}(1)$ by Proposition 1. 
Proof of Lemma 2. Note that $V(k)$ is described in the following manner:

$$
V(k)=\sum_{h=1}^{k-1}\left[\left(P\left(\alpha_{h}^{*}\right)-c-\rho\right)\left(\prod_{\ell=1}^{h} \alpha_{\ell}^{*}\right)\right]+P\left(\alpha_{k}^{*}\right) \prod_{\ell=1}^{k} \alpha_{\ell}^{*}
$$

where $\alpha_{h}^{*}=\gamma^{*}(k-h+1)$ for all $h=1, \ldots, k$. Let's look at the monopoly problem with $k+1$ consumers. Consider the following policy for the firm: set the same purchase probabilities for the first $k$ consumers as in the $k$-consumer problem (i.e., $\alpha_{h}=\alpha_{h}^{*}$ for all $h=1, \ldots, k$ ) and make the $k$ th consumer just willing to make a referral to the last $(k+1)$ th consumer (by setting the expected referral benefit equal to the referral cost: $\left.\alpha_{k+1}^{*} r_{k+1}^{*}=\rho\right)$. The monopoly profit under such a policy is

$$
\Pi\left(\alpha_{k+1} ; \alpha_{1}^{*}, \ldots, \alpha_{k}^{*}\right)=V(k)+\left(\alpha_{k+1} P\left(\alpha_{k+1}\right)-c-\rho\right)\left(\prod_{\ell=1}^{k} \alpha_{\ell}^{*}\right) .
$$

Note that $\prod_{\ell=1}^{k} \alpha_{\ell}^{*}$ is the unconditional probability that the $k$ th consumer purchases the product, and the firm pays $\rho$ to let her make a referral. Since the maximum $V$ is achieved with $\alpha_{k+1}=\alpha^{m}$, we have

$$
\widetilde{\Pi}=\max _{\alpha_{k+1}} \Pi\left(\alpha_{k+1} ; \alpha_{1}^{*}, \ldots, \alpha_{k}^{*}\right)=V(k)+\left(\alpha^{m} P\left(\alpha^{m}\right)-c-\rho\right)\left(\prod_{\ell=1}^{k} \alpha_{\ell}^{*}\right)>V(k),
$$

if and only if $\pi^{m}=\alpha^{m}\left(p^{m}-c\right)>\rho$. Thus, we have $\widetilde{\Pi}>V(k)$. Since $V(k+1) \geq \widetilde{\Pi}$, it follows that $V(k+1)>V(k)$ when $\pi^{m}>\rho$. $\square$

Proof of Proposition 2. From Lemma 2 and Corollary 1, we know that $\alpha_{1}^{*}>\alpha_{2}^{*}>\ldots>$ $\alpha_{n}^{*}=\alpha^{m}>0$ and $p=p^{m}$. From $(7), \alpha_{k}^{*} r_{k}^{*}=p-P\left(\alpha_{k-1}^{*}\right)+\rho$ for $k=2, \ldots, n$, and since $\alpha_{1}^{*}>\alpha_{2}^{*}>\ldots>\alpha_{n}^{*}$, we conclude that $\alpha_{2}^{*} r_{2}^{*}>\alpha_{3}^{*} r_{3}^{*}>\ldots>\alpha_{n}^{*} r_{n}^{*}>\rho . \square$

Proof of Proposition 3. We prove the first statement by induction. Since $\gamma^{*}(1)=$ $\alpha^{m}$ and $V(1)=\pi^{m}, \frac{d V(1)}{d c}=-\gamma^{*}(1)=-\alpha_{n}^{*}=-\alpha^{m}<0$. From (10), we have $\frac{d V(k)}{d c}=$ 
$-\gamma^{*}(k)\left(1-\frac{d V(k-1)}{d c}\right)$ by the envelope theorem for $k=2, \ldots, n$. Since $\gamma^{*}(k)=\alpha_{n-k+1}^{*}>0$, it follows that for any $k=2, \ldots, n$, if $\frac{d V(k-1)}{d c}<0$, then $\frac{d V(k)}{d c}<0$. Hence, $\frac{d V(k)}{d c}<0$ for $k=1, \ldots, n$. The first-order condition for $(11)$ is

$$
P\left(\gamma^{*}(k)\right)-c-\rho+V(k-1)+\gamma^{*}(k) P^{\prime}\left(\gamma^{*}(k)\right)=0 .
$$

Totally differentiating the above, we obtain

$$
\left(2 P^{\prime}\left(\gamma^{*}(k)\right)+\gamma^{*}(k) P^{\prime \prime}\left(\gamma^{*}(k)\right)\right) \frac{d \gamma^{*}(k)}{d c}=1-\frac{d V(k-1)}{d c}>0 .
$$

From the second-order condition for profit maximization, $2 P^{\prime}\left(\gamma^{*}(k)\right)+\gamma^{*}(k) P^{\prime \prime}\left(\gamma^{*}(k)\right)<0$ holds, and we conclude that $\frac{d \alpha_{n-k+1}^{*}}{d c}=\frac{d \gamma^{*}(k)}{d c}<0$ for $k=1, \ldots, n$.

We use similar arguments to derive the comparative statics result for referral cost $\rho$. First, $\frac{d V(1)}{d \rho}=0$ and $\frac{d V(2)}{d \rho}=-\gamma^{*}(2)<0$. From (10), $\frac{d V(k)}{d \rho}=-\gamma^{*}(k)\left(1-\frac{d V(k-1)}{d \rho}\right)$. Since $\gamma^{*}(k)=$ $\alpha_{n-k+1}^{*}>0$, it follows that if $\frac{d V(k-1)}{d \rho}<0$, then $\frac{d V(k)}{d \rho}<0$ for $k=2, \ldots, n$. Hence, $\frac{d V(1)}{d \rho}=0$ and $\frac{d V(k)}{d \rho}<0$ for $k=2, \ldots, n$. From (11), we obtain $\left(2 P^{\prime}\left(\gamma^{*}(k)\right)+\gamma^{*}(k) P^{\prime \prime}\left(\gamma^{*}(k)\right)\right) \frac{d \gamma^{*}(k)}{d \rho}=$ $1-\frac{d V(k-1)}{d \rho}>0$, and we can conclude that $\frac{d \alpha_{n}^{*}}{d \rho}=\frac{d \gamma^{*}(1)}{d \rho}=0$ and $\frac{d \alpha_{n-k+1}^{*}}{d \rho}=\frac{d \gamma^{*}(k)}{d \rho}<0$ for $k=2, \ldots, n$.

From Proposition 2, the optimal price is $p^{*}=p^{m}$, and therefore it decreases in production cost $c$ but is independent of referral cost $\rho$. Finally, from (7), $\alpha_{k}^{*} r_{k}^{*}-\rho=p^{m}-P\left(\alpha_{k-1}^{*}\right)$. Hence, $\frac{d\left(\alpha_{k}^{*} r_{k}^{*}-\rho\right)}{d \rho}=-P^{\prime}\left(\alpha_{k-1}^{*}\right) \frac{d \alpha_{k-1}^{*}}{d \rho}<0$.

Proof of Proposition 4. To see that $r_{k}^{*}<r_{k-1}^{*}$ holds for $k=2, \ldots, n$, in the case of the uniform distribution of values, note that the profit-maximizing solution in this case is $\gamma(k)=$ $\arg \max _{\alpha}\{\alpha[1-\alpha-c-\rho+V(k-1)]\}$ for all $k=2, \ldots$, and $\gamma(1)=\alpha_{n}^{*}=\frac{1-c}{2}$. Solving this, we have $\gamma(k)=\frac{1}{2}(1-c-\rho+V(k-1))$. Thus, $V(k)=\gamma(k)[1-\gamma(k)-c-\rho+V(k-1)]=$ 
$\left\{\frac{1}{2}(1-c-\rho+V(k-1))\right\}^{2}=(\gamma(k))^{2}$ for all $k=2, \ldots, n$. We find that $\gamma(k)=\frac{1}{2}\left(1-c-\rho+(\gamma(k-1))^{2}\right)$

for all $k=2, \ldots, n$ with $\gamma(1)=\alpha_{n}^{*}=\frac{1-c}{2}$. From (7), we have

$$
\begin{aligned}
r_{k}^{*} & =\frac{P\left(\alpha_{n}^{*}\right)-P\left(\alpha_{k-1}^{*}\right)+\rho}{\alpha_{k}^{*}} \\
& =\frac{P(\gamma(1))-P(\gamma(n-k+2))+\rho}{\gamma(n-k+1)} \\
& =\frac{1-\frac{1-c}{2}-1+\frac{1}{2}\left(1-c-\rho+(\gamma(n-k+1))^{2}\right)+\rho}{\gamma(n-k+1)} \\
& =\frac{\rho}{2 \gamma(n-k+1)}+\frac{\gamma(n-k+1)}{2}=\frac{\rho}{2 \alpha_{k}^{*}}+\frac{\alpha_{k}^{*}}{2} .
\end{aligned}
$$

As is easily verified, $r_{k}^{*}$ is increasing in $\alpha_{k}^{*}$ as long as $\left(\alpha_{k}^{*}\right)^{2}>\rho$. This inequality holds for any $k \leq n$ because $\pi^{m}=\left(\alpha_{n}^{*}\right)^{2}>\rho$ and $V(1)=\pi^{m}<V(n-k+1)=\left(\alpha_{k}^{*}\right)^{2}$ for any $k<n$. Since by Proposition $2, \alpha_{k}^{*}$ is a decreasing sequence, so is $r_{k}$. Thus, we have $r_{2}^{*}>r_{3}^{*}>\ldots>r_{n}^{*}>0$. By Proposition $3, \frac{d \alpha_{k}^{*}}{d c}<0$ for $k=1, \ldots, n$ and since $\frac{d r_{k}^{*}}{d c}=\frac{\alpha_{k}^{* 2}-\rho}{2 \alpha_{k}^{* 2}} \frac{d \alpha_{k}^{*}}{d c}$, it follows that referral fees $r_{k}^{*}$ are decreasing in production cost as well, $\frac{d r_{k}^{*}}{d c}<0$ for $k=2, \ldots, n$. Together, these results imply that the expected referral payments $r_{k}^{*} \alpha_{k}^{*}$ are decreasing in production cost for any $k=2, \ldots, n . \square$ 


\section{Appendix B: The Second-Best Problem}

Following Jun and Kim (2008), let us consider the case where the firm has to set a common referral fee and price to all consumers, regardless of whether they are early or late adopters. Jun and Kim show that when the second-to-last consumer has a strictly positive referral benefit, $r(1-F(p))>\rho$ (their referral condition $\mathrm{RC}$ ), the earlier a consumer is located in the chain, the higher is her probability of purchasing the product $\alpha_{1}>\alpha_{2}>\ldots>\alpha_{n}$ (their Proposition 1). This result further implies effective price discrimination among consumers according to their position in the referral chain: although the firm charges a common price $p$ and pays a referral fee $r$ to all consumers, the firm effectively discriminates in favor of consumers located earlier in the chain because these consumers obtain a higher expected benefit from making a referral. ${ }^{10}$

We take a closer look at the optimal strategy of the firm. In particular, we examine the possibility of a stationary outcome being optimal. We allow for referral equilibrium to be consistent with a binding referral condition $r(1-F(p))=\rho$ by assuming that when indifferent, consumers make referrals. We show that the firm's profit can be improved by increasing both $p$ and $r$ in a right proportion starting from the optimal stationary outcome, implying that the stationary outcome is not even a local maximum for any finite $n$ (Proposition B1). This result strongly justifies Jun and Kim's analysis and also implies that at least for large $n$, the optimal solution is perhaps very close to the stationary outcome.

Denote by $\alpha_{k}$ the probability that consumer $i$ buys the product conditional on being

\footnotetext{
${ }^{10}$ Consumer $k$ 's purchase probability $\alpha_{k}$ depends on consumer $(k+1)$ 's purchase probability $\alpha_{k+1}$ since consumer $k$ takes the expected net benefit from referral $r \alpha_{k+1}-\rho$ into account when she makes her purchase decision: $\left.\alpha_{k}=1-F\left(p+r \alpha_{k+1}-\rho\right)\right)$.
} 
introduced to it, $k=1, \ldots, n$. The firm chooses a policy $(p, r)$ to maximize its profits

$$
\begin{aligned}
\hat{\Pi}(p, r) & =\left(p-r \alpha_{2}-c\right) \alpha_{1}+\left(p-r \alpha_{3}-c\right) \alpha_{1} \alpha_{2}+\ldots \\
& +\left(p-r \alpha_{n}-c\right) \alpha_{1} \cdots \alpha_{n-1}+(p-c) \alpha_{1} \cdots \alpha_{n}
\end{aligned}
$$

where $\alpha_{1}, \ldots, \alpha_{n}$ are determined by $(p, r)$ as follows: $\alpha_{n}=D(p)=1-F(p) \geq 0$ and $\alpha_{k}=D\left(p-\alpha_{k+1} r+\rho\right) \geq 0$ for $k=1, \ldots, n-1$. Denote by $P(\alpha)=D^{-1}(\alpha)$ the standard inverse demand function. We assume that the profit function without referrals, $\pi(\alpha) \equiv$ $\alpha(P(\alpha)-c)$, is concave.

Assuming $r \alpha_{k} \geq \rho$ for $k=2, \ldots, n, \alpha_{1}, \ldots, \alpha_{n}$, are determined by the following system of equations:

$$
\begin{gathered}
P\left(\alpha_{n}\right)=p \\
P\left(\alpha_{n-1}\right)=p-r \alpha_{n}+\rho \\
\cdots \\
P\left(\alpha_{1}\right)=p-r \alpha_{2}+\rho,
\end{gathered}
$$

and $\alpha_{1} \geq \ldots \geq \alpha_{n}$. Suppose that the referral condition is binding for the $k$ th consumer: $r \alpha_{k+1}=\rho$ for some $k=1, \ldots, n-1$. Then, $P\left(\alpha_{k}\right)=p$, and we have $P\left(\alpha_{1}\right)=P\left(\alpha_{2}\right)=$ $\ldots=P\left(\alpha_{n}\right)=p$ and $\alpha_{1}=\alpha_{2}=\ldots=\alpha_{n}$. This is a stationary outcome, for which consumer referral conditions are all binding: $r \alpha_{k+1}=\rho$ for all $k=1, \ldots, n-1$. We will show that this outcome is not locally optimal.

The firm's profit can be written in terms of $\alpha_{k}$ s only:

$$
\begin{aligned}
\Pi\left(\alpha_{1}, \ldots, \alpha_{n-1}, \alpha_{n} ; n\right) & =\alpha_{1}\left(P\left(\alpha_{1}\right)-\rho-c\right)+\alpha_{1} \alpha_{2}\left(P\left(\alpha_{2}\right)-\rho-c\right)+\ldots \\
& +\alpha_{1} \cdots \alpha_{n-1}\left(P\left(\alpha_{n-1}\right)-c-\rho\right)+\alpha_{1} \cdots \alpha_{n}\left(P\left(\alpha_{n}\right)-c\right)
\end{aligned}
$$


where $\left(\alpha_{1}, \ldots, \alpha_{n}\right)$ is a solution to system (23). Under the stationary outcome $\alpha_{1}=\ldots=$ $\alpha_{n-1}=\alpha_{n}=\alpha$, the monopoly profit when there are $n \geq 1$ consumers can be written as

$$
\Pi(\alpha, \alpha, \ldots, \alpha ; n)=A_{n}(\alpha)(\pi(\alpha)-\rho)+\rho,
$$

where

$$
A_{n}(\alpha) \equiv 1+\alpha+\alpha^{2}+\ldots+\alpha^{n-1}=\frac{1-\alpha^{n}}{1-\alpha}
$$

and $\pi(\alpha)=\alpha(P(\alpha)-c)$.

Denote the optimal stationary policy for an $n$-consumer chain by $\beta(n) \equiv \arg \max _{\alpha} \Pi(\alpha, \alpha, \ldots, \alpha ; n)$. Theorem 2 states that $\beta(n)$ cannot be a local maximum for small $\rho$.

Proposition B1. The optimal stationary policy $\beta(n)$ is not the optimal policy if $\pi(\beta(n))>\rho$.

To prove Proposition B1, we will show that the firm's profit is locally improvable (starting from $\beta(n))$ by choosing an appropriate policy change $(d p, d r) \gg 0$. We first provide a sketch of the proof of Proposition B1. First, in Lemma B1, we investigate the properties of the optimal stationary policy $\beta(n)$. Then, we look at the profit function evaluated at the optimal stationary policy $\alpha_{1}=\ldots=\alpha_{n-1}=\alpha_{n}=\beta(n)$. We show that there is some $M(1 \leq M<n)$ such that profits increase with purchase probability for consumers located before $M$ and decrease with purchase probability for consumers located after $M:\left.\frac{\partial \Pi}{\partial \alpha_{k}}\right|_{\alpha=\beta(n)}>0$ for all $k<M$ and $\left.\frac{\partial \Pi}{\partial \alpha_{k}}\right|_{\alpha=\beta(n)}<0$ for all $k>M$ (Lemma B2). We then show that there exists a policy change $d \Delta=(d p, d r) \gg 0$ such that for any $M(1 \leq M<n)$ the probability of buying increases for consumers located before $M$ and decreases for consumers located after $M$. We prove this by showing that, starting at $\alpha_{1}=\ldots=\alpha_{n}=\alpha$, if $\alpha_{n}$ decreases while $\alpha_{M}$ is kept constant, $d \alpha_{k}>0$ for all $k<M$ and $d \alpha_{k}<0$ for all $k>M$ (Lemma B3). Using Lemmas B2 and B3, we conclude that the optimal stationary policy is not a local maximum. 
We prove Proposition B1 by using a sequence of lemmas.

\section{Lemma B1.}

(i) For all $n$ and all $\alpha$ such that $\pi(\alpha)-\rho>0, \Pi(\alpha, \alpha, \ldots, \alpha ; n+1)>\Pi(\alpha, \alpha, \ldots, \alpha ; n)$.

(ii) The optimal stationary solution $\beta(n) \equiv \arg \max _{\alpha} \Pi(\alpha, \alpha, \ldots, \alpha ; n)$ satisfies the following condition:

$$
\pi^{\prime}(\beta(n))=-\frac{A_{n}^{\prime}(\beta(n))}{A_{n}(\beta(n))}(\pi(\beta(n))-\rho) .
$$

(iii) Suppose $\pi(\beta(n))-\rho>0$. Then, $\beta(n)>\beta(n-1)>\ldots>\beta(1)=\arg \max _{\tilde{\alpha}} \pi(\tilde{\alpha})$.

Proof. From (25), the difference in profits from $(n+1)$ - and $n$-consumer chains is

$$
\Delta^{n}(\alpha) \equiv \Pi(\alpha, \alpha, \ldots, \alpha ; n+1)-\Pi(\alpha, \alpha, \ldots, \alpha ; n)=\alpha^{n}(\pi(\alpha)-\rho)
$$

Hence, $\Delta^{n}(\alpha)>0$ if $\pi(\alpha)-\rho>0$. This proves (i).

The optimal policy $\alpha=\beta(n)$ is implicitly defined by the first-order condition

$$
\frac{d \Pi(\alpha, \alpha, \ldots, \alpha ; n)}{d \alpha}=A_{n}^{\prime}(\alpha)(\pi(\alpha)-\rho)+A_{n}(\alpha) \pi^{\prime}(\alpha)=0
$$

This proves (ii).

Finally, using (27), we find that at $\alpha=\beta(n)$

$$
\begin{aligned}
\frac{d \Delta^{n}}{d \alpha} & =n \alpha^{n-1}(\pi(\alpha)-\rho)+\alpha^{n} \pi^{\prime}(\alpha) \\
& =\alpha^{n-1}(\pi(\alpha)-\rho)\left(\frac{n A_{n}(\alpha)-\alpha A_{n}^{\prime}(\alpha)}{A_{n}(\alpha)}\right)>0 .
\end{aligned}
$$

The last inequality holds because

$$
\begin{aligned}
n A_{n}(\alpha)-\alpha A_{n}^{\prime}(\alpha) & =n\left(1+\alpha+\alpha^{2}+\ldots+\alpha^{n-1}\right)-\alpha\left(1+2 \alpha+\ldots+(n-1) \alpha^{n-2}\right) \\
& =n+(n-1) \alpha+(n-2) \alpha^{2}+\ldots+\alpha^{n-1}>0 .
\end{aligned}
$$


Hence, if $\alpha=\beta(n)>0$ and $\pi(\beta(n))-\rho>0$, then $\beta(n+1)>\beta(n)$. Since $\beta(1)=$ $\alpha^{m}>0$, it follows that $\pi^{\prime}(\alpha)<0$ for all $\alpha>\beta(1)$. Thus, $\pi(\beta(1))>\pi(\beta(2))>\ldots>\pi(\beta(n))$ holds, and we conclude that $\beta(1)>\beta(2)>\ldots>\beta(n)$ if $\pi(\beta(n))-\rho>0 . \square$

Notice that the profit $\Pi\left(\alpha_{1}, \alpha_{2}, \ldots, \alpha_{n} ; n\right)$ in equation (24) can be defined recursively:

$$
\begin{gathered}
\Pi\left(\alpha_{n} ; 1\right)=\pi\left(\alpha_{n}\right)=\alpha_{n}\left(P\left(\alpha_{n}\right)-c\right) \\
\Pi\left(\alpha_{n-1}, \alpha_{n} ; 2\right)=\alpha_{n-1}\left(P\left(\alpha_{n-1}\right)-c-\rho\right)+\alpha_{n-1} \Pi\left(\alpha_{n} ; 1\right) \\
\Pi\left(\alpha_{n-2}, \alpha_{n-1}, \alpha_{n} ; 3\right)=\alpha_{n-2}\left(P\left(\alpha_{n-2}\right)-c-\rho\right)+\alpha_{n-2} \Pi\left(\alpha_{n-1}, \alpha_{n} ; 2\right) \\
\ldots \\
\Pi\left(\alpha_{1}, \alpha_{2}, \ldots, \alpha_{n} ; n\right)=\alpha_{1}\left(P\left(\alpha_{1}\right)-c-\rho\right)+\alpha_{1} \Pi\left(\alpha_{2}, \ldots, \alpha_{n} ; n-1\right)
\end{gathered}
$$

Using these formulas, we prove the following result.

Lemma B2. Suppose that $\pi(\beta(n))-\rho>0$ holds. At $\alpha_{1}=\ldots=\alpha_{n}=\beta(n)$, (i) $\frac{\partial \Pi}{\partial \alpha_{n}}<0$ and $\frac{\partial \Pi}{\partial \alpha_{1}}>0$; (ii) there exists $M$ such that $\frac{\partial \Pi}{\partial \alpha_{k}}>0$ for any $k<M$ and $\frac{\partial \Pi}{\partial \alpha_{k}}<0$ for any $k>M$.

Proof. The marginal profits with respect to buying probabilities $\alpha_{1}, \ldots, \alpha_{n}$ are

$$
\begin{aligned}
\frac{1}{\alpha_{1} \alpha_{2} \alpha_{3} \cdots \alpha_{n-1}} \frac{\partial \Pi}{\partial \alpha_{n}} & =\pi^{\prime}\left(\alpha_{n}\right) \\
\frac{1}{\alpha_{1} \alpha_{2} \alpha_{3} \cdots \alpha_{n-2}} \frac{\partial \Pi}{\partial \alpha_{n-1}} & =\pi^{\prime}\left(\alpha_{n-1}\right)+\Pi\left(\alpha_{n} ; 1\right)-\rho \\
\frac{1}{\alpha_{1} \alpha_{2} \alpha_{3} \cdots \alpha_{n-3}} \frac{\partial \Pi}{\partial \alpha_{n-2}} & =\pi^{\prime}\left(\alpha_{n-2}\right)+\Pi\left(\alpha_{n-1}, \alpha_{n} ; 2\right)-\rho \\
& \cdots \\
\frac{\partial \Pi}{\partial \alpha_{1}} & =\pi^{\prime}\left(\alpha_{1}\right)+\Pi\left(\alpha_{2}, \ldots, \alpha_{n} ; n-1\right)-\rho .
\end{aligned}
$$


Thus, at $\alpha_{1}=\alpha_{2}=\ldots=\alpha_{n}=\alpha$,

$$
\begin{aligned}
\frac{\partial \Pi}{\partial \alpha_{n}} & =\alpha^{n-1} \pi^{\prime}(\alpha) \\
\frac{\partial \Pi}{\partial \alpha_{n-1}} & =\alpha^{n-2}\left(\pi^{\prime}(\alpha)+\Pi(\alpha ; 1)-\rho\right) \\
& \ldots \\
\frac{\partial \Pi}{\partial \alpha_{1}} & =\pi^{\prime}(\alpha)+\Pi(\alpha, \ldots, \alpha ; n-1)-\rho
\end{aligned}
$$

Note that $\frac{\partial \Pi}{\partial \alpha_{n}}=\alpha^{n-1} \pi^{\prime}(\alpha)<0$ at $\beta(n)>\beta(1)=\alpha^{m}$ by Lemma B1. By assumption, $\Pi(\alpha ; 1)=\pi(\beta(n))>\rho$, and by Lemma $\mathrm{B} 1, \Pi(\alpha, \ldots, \alpha ; k)$ is increasing in $k$. Hence, if for some $\ell, \frac{\partial \Pi}{\partial \alpha_{\ell}}>0$, then $\frac{\partial \Pi}{\partial \alpha_{k}}>0$ for any $k<\ell$, and if $\frac{\partial \Pi}{\partial \alpha_{\ell}}<0$, then $\frac{\partial \Pi}{\partial \alpha_{k}}<0$ for any $k>\ell$.

In the following, we will show that for $\alpha=\beta(n)>0$, there exists $M$ such that $\frac{\partial \Pi}{\partial \alpha_{k}}>0$ for any $k<M$ and $\frac{\partial \Pi}{\partial \alpha_{k}}<0$ for any $k>M$. Recall

$$
A_{k}(\alpha)=1+\alpha+\alpha^{2}+\ldots+\alpha^{k-1}=\frac{1-\alpha^{k}}{1-\alpha} .
$$

For $k \leq n-1$, we have

$$
\frac{\partial \Pi}{\partial \alpha_{k}}=\alpha^{k-2}\left(\pi^{\prime}(\alpha)+\Pi(\alpha, \ldots, \alpha ; n-k+1)-\rho\right)
$$

where

$$
\Pi(\alpha, \alpha, \ldots, \alpha ; n-k+1)=A_{n-k+1}(\alpha)(\pi(\alpha)-\rho)+\rho
$$

Hence, $\frac{\partial \Pi}{\partial \alpha_{k}}>0$ as long as

$$
\pi^{\prime}(\alpha)+A_{n-k+1}(\alpha)(\pi(\alpha)-\rho)>0
$$

Plugging in the expression for the optimal $\pi^{\prime}(\alpha)$ from Lemma B1 and assuming $\pi(\beta(n))-$ $\rho>0$, the inequality is equivalent to

$$
A_{n-k+1}(\alpha) A_{n}(\alpha)>A_{n}^{\prime}(\alpha)
$$


which is equivalent to

$$
\frac{1-\alpha^{n-k+1}}{1-\alpha} \frac{1-\alpha^{n}}{1-\alpha}>\frac{\partial\left(\frac{1-\alpha^{n}}{1-\alpha}\right)}{\partial \alpha}=\frac{1}{(1-\alpha)^{2}}\left(1-\alpha^{n}-n \alpha^{n-1}+n \alpha^{n}\right) .
$$

Thus, we conclude that $\frac{\partial \Pi}{\partial \alpha_{k}}>0$ holds if and only if

$$
\left[n \alpha^{n-1} \frac{1-\alpha}{1-\alpha^{n}}-\alpha^{n-k+1}\right]>0 .
$$

The expression in the brackets is strictly decreasing in $k$. Note that for $k=1,\left[n \alpha^{n-1} \frac{1-\alpha}{1-\alpha^{n}}-\alpha^{n}\right]=$ $\left(n \frac{1-\alpha}{1-\alpha^{n}}-\alpha\right) \alpha^{n-1}>0$ because $n(1-\alpha)-\alpha\left(1-\alpha^{n}\right)>n(1-\alpha)-\alpha(1-\alpha)=(n-\alpha)(1-\alpha)>$ 0. Hence, $\frac{\partial \Pi}{\partial \alpha_{1}}>0$. Since $\frac{\partial \Pi}{\partial \alpha_{n}}=\alpha^{n-1} \pi^{\prime}(\alpha)<0$, there exists $M(1 \leq M<n)$ such that $\frac{\partial \Pi}{\partial \alpha_{k}}>0$ for any $k<M$ and $\frac{\partial \Pi}{\partial \alpha_{k}}<0$ for any $k>M$ at $\alpha=\beta(n)$.

In Lemma B3, we describe the effects of a policy change $(d p, d r) \gg 0$ at a stationary outcome $\alpha_{1}=\ldots=\alpha_{n}=\alpha$.

Lemma B3. Consider a policy of increasing $p$ and $r$, starting at $\alpha_{1}=\ldots=\alpha_{n}=\alpha$. For any $M(1 \leq M<n)$, there is a policy change $(d p, d r) \gg 0$ such that $d \alpha_{k}>0$ for all $k<M$ and $d \alpha_{k}<0$ for all $k>M$.

Proof. Totally differentiating equations (23) and evaluating at $\alpha_{1}=\ldots=\alpha_{n}=\alpha$, we have:

$$
\begin{aligned}
P^{\prime}(\alpha) d \alpha_{n} & =d p \\
P^{\prime}(\alpha) d \alpha_{n-1} & =(d p-\alpha d r)-r d \alpha_{n} \\
P^{\prime}(\alpha) d \alpha_{n-2} & =(d p-\alpha d r)-r d \alpha_{n-1} \\
\cdots & \\
P^{\prime}(\alpha) d \alpha_{1} & =(d p-\alpha d r)-r d \alpha_{2} .
\end{aligned}
$$


When $p$ is increasing $(d p>0)$, we necessarily have $d \alpha_{n}=\frac{1}{P^{\prime}(\alpha)} d p<0$.

Let $x \equiv \frac{r}{-P^{\prime}(\alpha)}>0$. We choose $(d p, d r) \gg 0$ such that $d \alpha_{M}=0$. From

$$
\begin{aligned}
& P^{\prime}(\alpha) d \alpha_{M} \\
& =(d p-\alpha d r)-r d \alpha_{M+1} \\
& =(d p-\alpha d r)(1+x)-r x d \alpha_{M+2} \\
& =(d p-\alpha d r)\left(1+x+\ldots+x^{n-M-1}\right)-r x^{n-M-1} d \alpha_{n} \\
& =(d p-\alpha d r)\left(1+x+\ldots+x^{n-M-1}\right)+x^{n-M} d p \\
& =d p\left(1+x+\ldots+x^{n-M}\right)-\alpha d r\left(1+x+\ldots+x^{n-M-1}\right)=0
\end{aligned}
$$

it follows that $d \alpha_{M}=0$ implies

$$
d p=\alpha d r \frac{1+x+\ldots+x^{n-M-1}}{1+x+\ldots+x^{n-M}}
$$

Similarly,

$$
P^{\prime}(\alpha) d \alpha_{k}=d p\left(1+x+\ldots+x^{n-k}\right)-\alpha d r\left(1+x+\ldots+x^{n-k-1}\right)
$$

Using (43),

$$
P^{\prime}(\alpha) d \alpha_{k}=\alpha d r\left[\frac{1+x+\ldots+x^{n-M-1}}{1+x+\ldots+x^{n-M}}\left(1+x+\ldots+x^{n-k}\right)-\left(1+x+\ldots+x^{n-k-1}\right)\right]
$$

Then, since $P^{\prime}(\alpha)<0$ and $d r>0, d \alpha_{k}>0$ if and only if

$$
\frac{1+x+\ldots+x^{n-M-1}}{1+x+\ldots+x^{n-M}}<\frac{1+x+\ldots+x^{n-k-1}}{1+x+\ldots+x^{n-k}}
$$

This inequality holds whenever $k<M$ because $\frac{1+x+\ldots+x^{a-1}}{1+x+\ldots+x^{a}}=\frac{1-x^{a}}{1-x^{a+1}}$ and $\frac{\partial\left(\frac{1-x^{a}}{1-x^{a+1}}\right)}{\partial a}=$ $x^{a} \frac{\ln x}{\left(1-x^{a+1}\right)^{2}}(x-1)>0$. Similarly, $d \alpha_{k}<0$ whenever $k>M$. $\square$ 
From Lemmas B2 and B3, we conclude that, assuming $\pi(\beta(n))>\rho$, the optimal stationary outcome $\beta(n)$ is not a local optimum for any finite $n$. This proves Proposition B1.

It follows from Proposition B1 that the optimal strategy $(p, r)$ is such that the referral condition is not binding for any consumer. This justifies the analysis of Jun and Kim (2008). As is known from Jun and Kim's Proposition 1, this implies that the firm effectively pricediscriminates by subsidizing consumer referrals and supporting a decreasing sequence of purchase probabilities, $\alpha_{1}>\ldots>\alpha_{n}$. 


\section{References}

[1] Simon P. Anderson and André de Palma. 2009. Information congestion. RAND Journal of Economics 40(4), 688-709.

[2] Maria Arbatskaya and Hideo Konishi. 2013. Consumer referrals. Department of Economics at Emory Working Paper 13-10.

[3] Andrea Galeotti and Sanjeev Goyal. 2009. Influencing the influences: a theory of strategic diffusion. RAND Journal of Economics 40(3), 509-532.

[4] Tackseung Jun and Jeong-Yoo Kim. 2008. A theory of consumer referral. International Journal of Industrial Organization 26 (3), 662-678. 


\section{FIGURES}

Figure 1. The Optimal Solution for the Chain of Length $n=5$

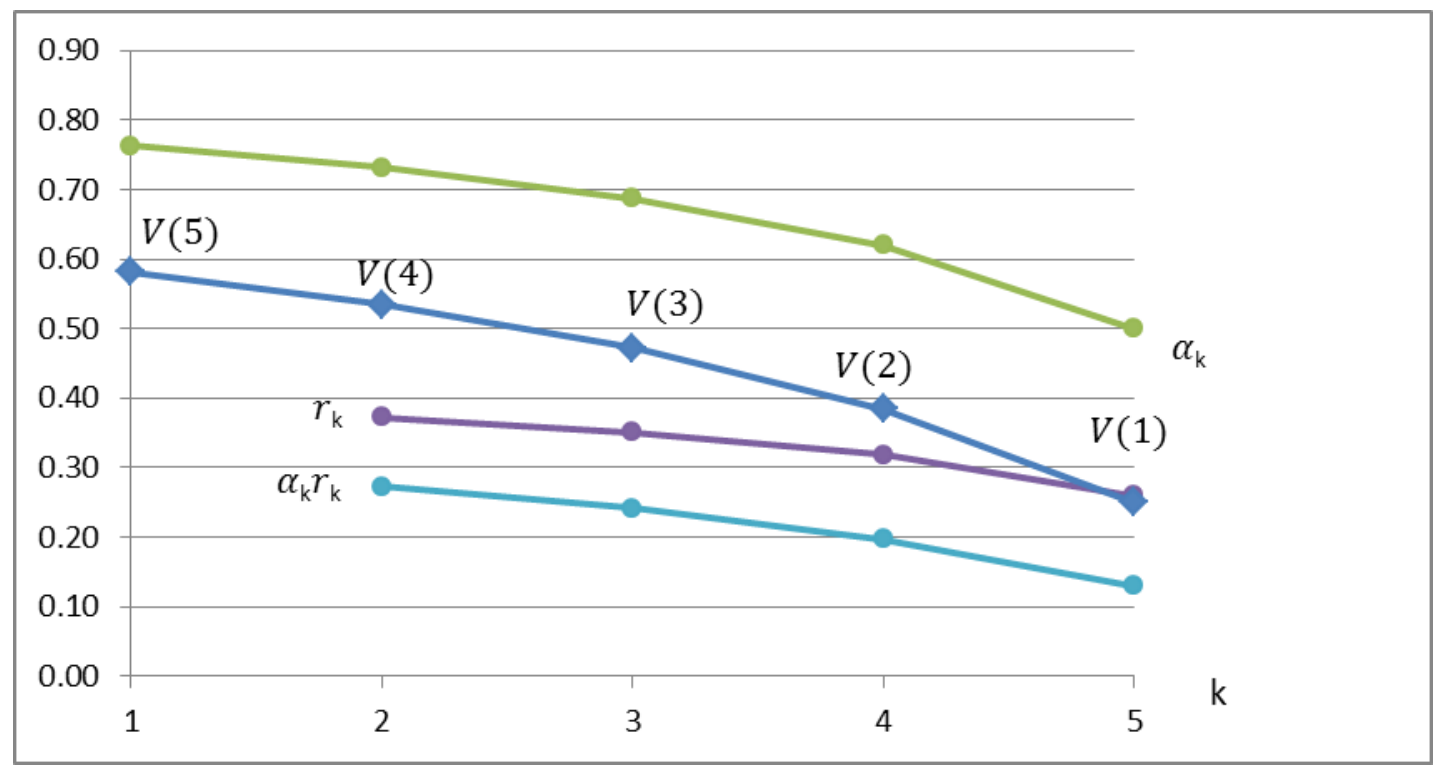

Notes: Purchase probability $\left(\alpha_{k}\right)$, referral fee $\left(r_{k}\right)$, the expected referral payments $\left(\alpha_{k} r_{k}\right)$ for the $k^{\text {th }}$ consumer in the chain, and the optimal profits starting from the $k$ th consumer (for the remaining chain of $n-k+1$ consumers), $V(n-k+1)$, are plotted for the uniform distribution of values, $n=5$, $\rho=0.01$, and $c=0.1$

Figure 2. Comparative Statics Results for Referral Cost

Panel A. Purchase Probabilities $\alpha_{1}$ through $\alpha_{5}$ as Functions of Referral Cost $\rho$.

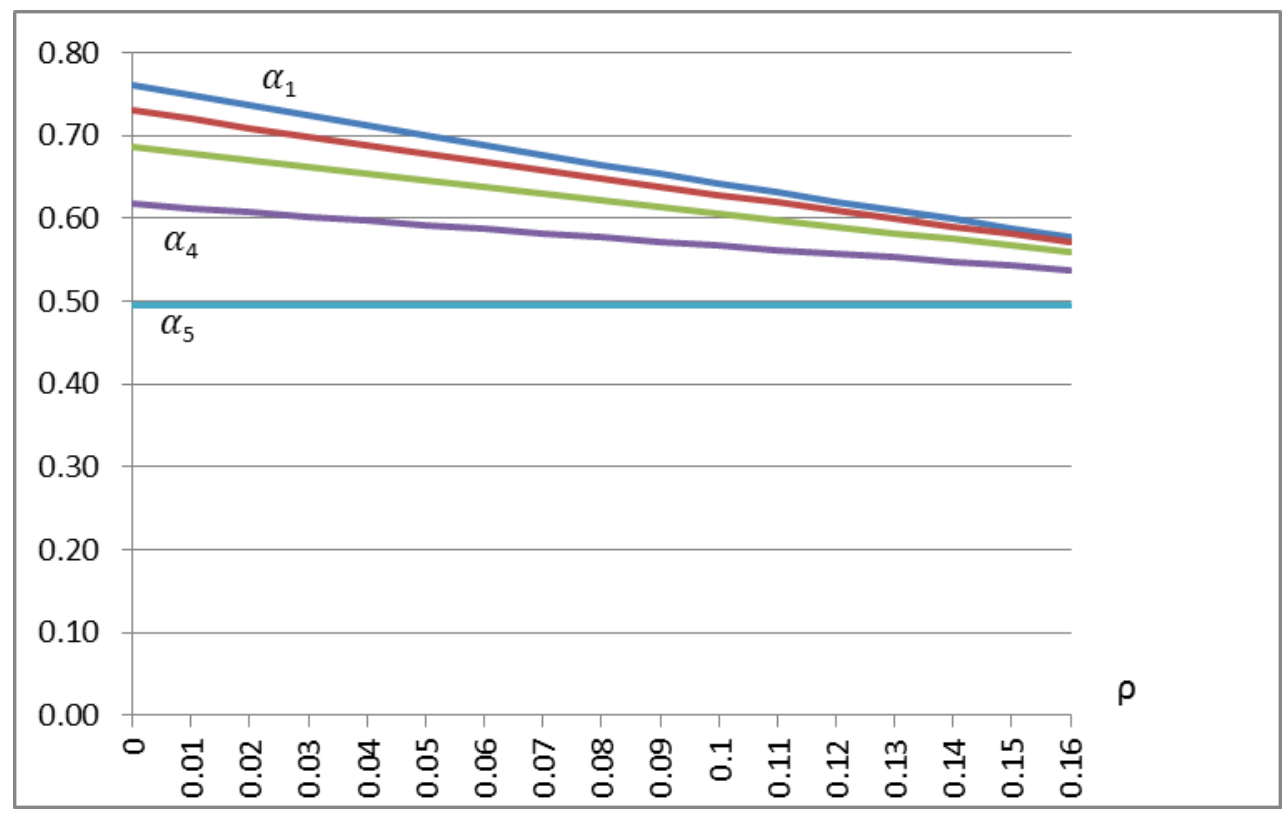


Panel B. Referral Fees $r_{2}$ through $r_{5}$ as Functions of Referral Cost $\rho$.

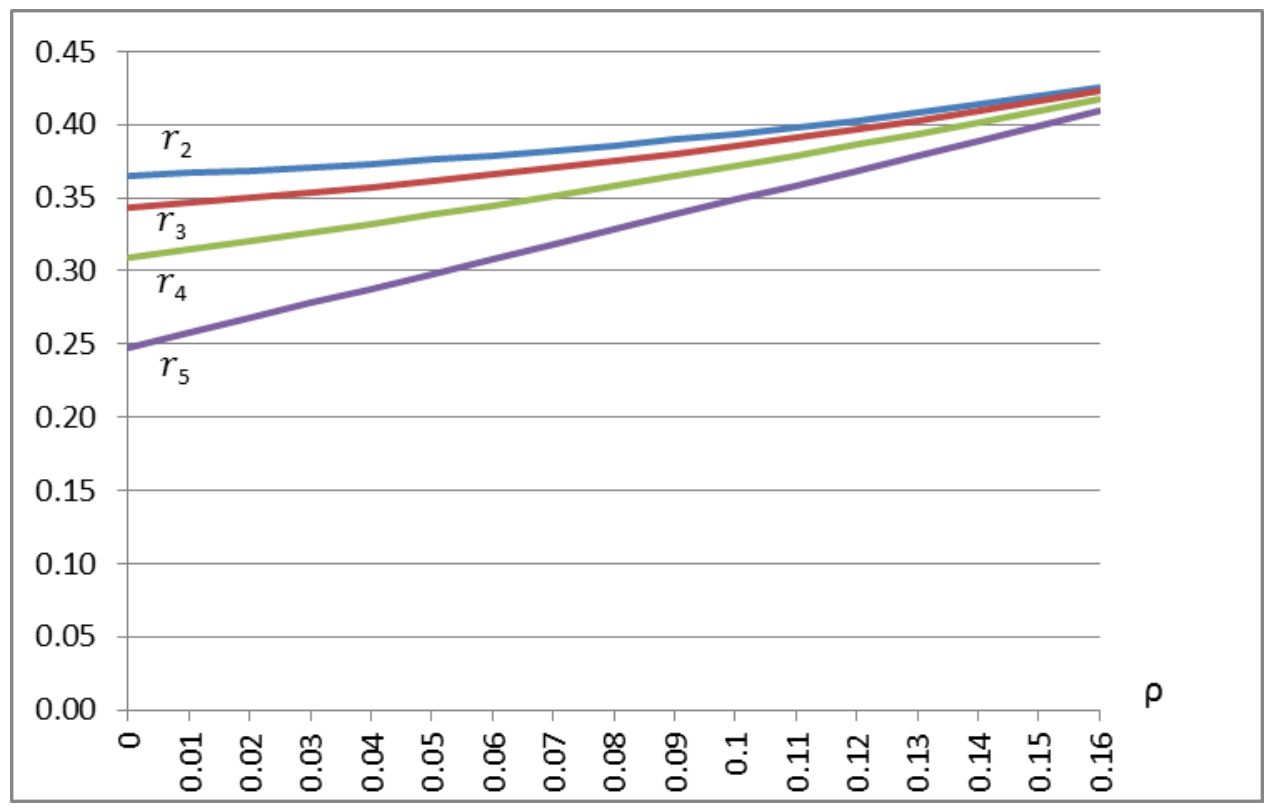

Panel C. Expected Referral Payments $\alpha_{2} r_{2}$ through $\alpha_{5} r_{5}$ as Functions of Referral Cost $\rho$.

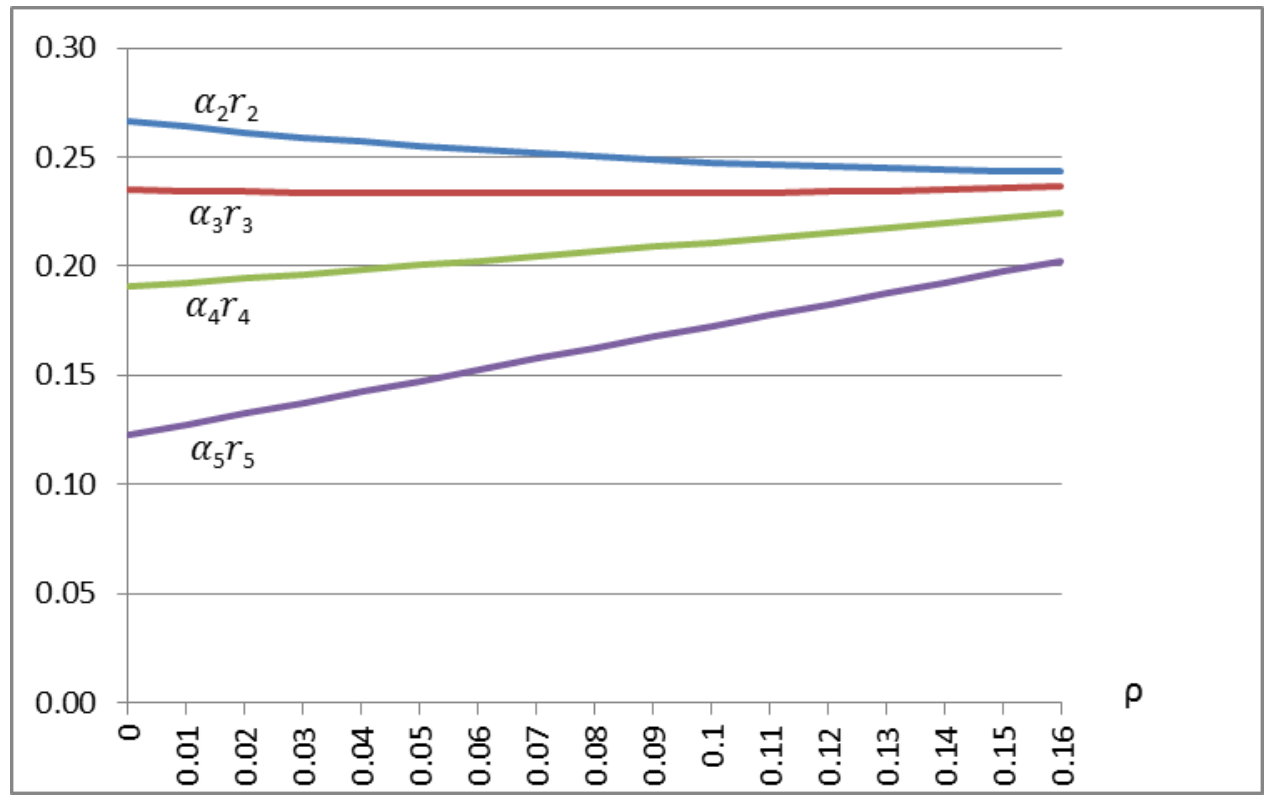

Notes: $\mathrm{n}=5$ and $\mathrm{c}=0.01$. 
Figure 3. Comparative Statics Results for Production Cost

Panel A. Purchase Probabilities $\alpha_{1}$ through $\alpha_{5}$ as Functions of Production Cost $c$.

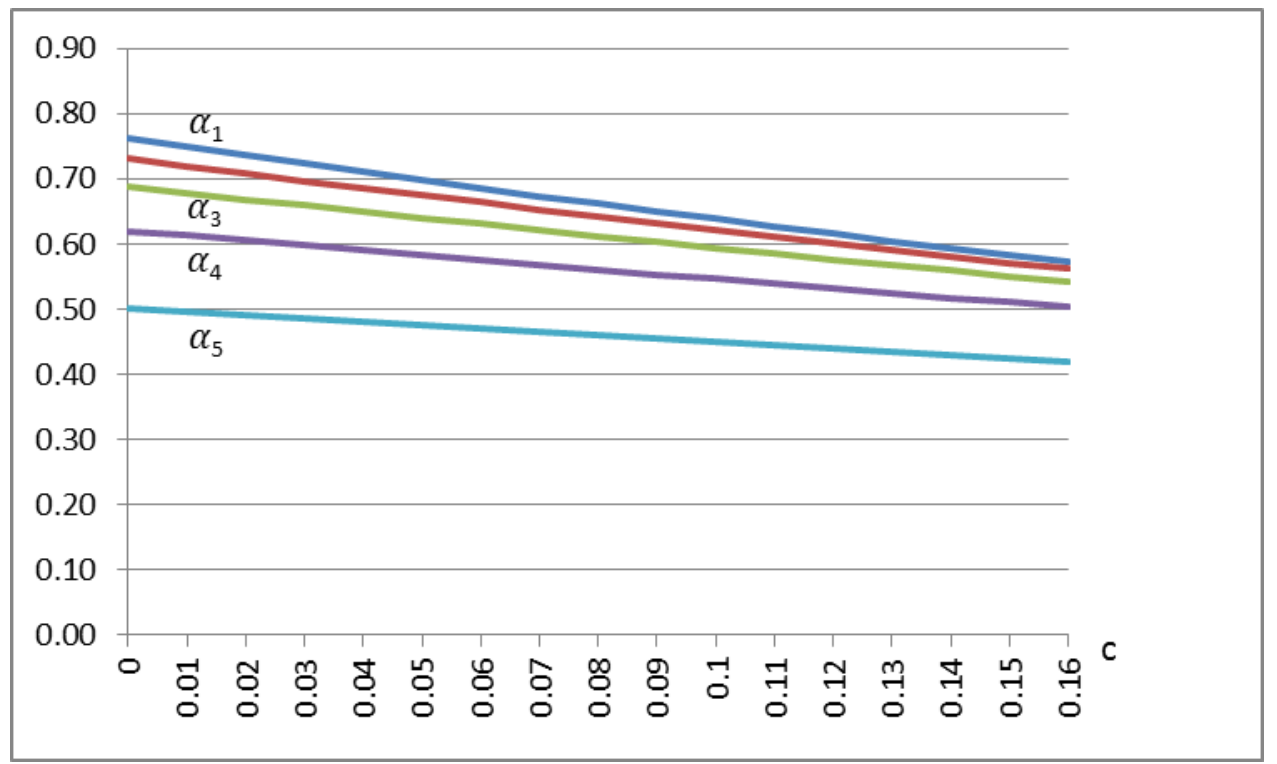

Panel B. Referral Fees $r_{2}$ through $r_{5}$ as Functions of Production Cost $c$.

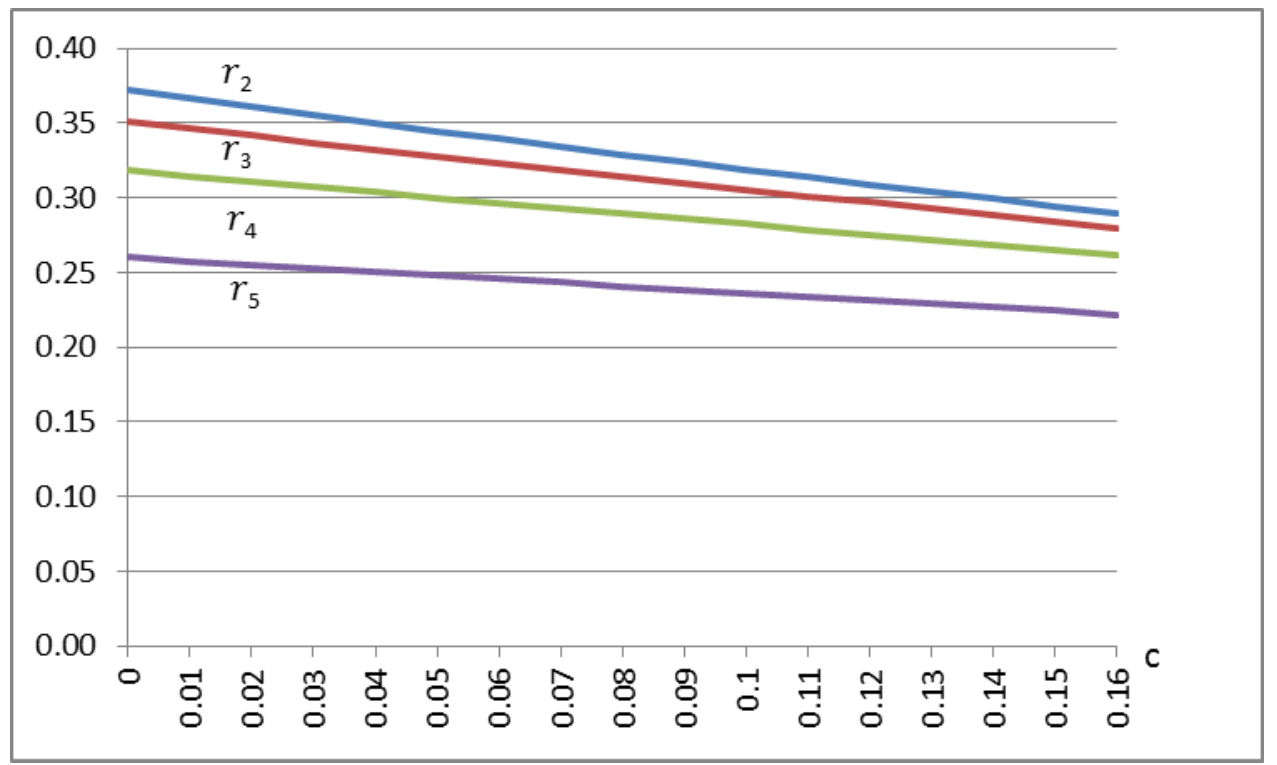


Panel C. Expected Referral Payments $\alpha_{2} r_{2}$ through $\alpha_{5} r_{5}$ as Functions of Production Cost $c$

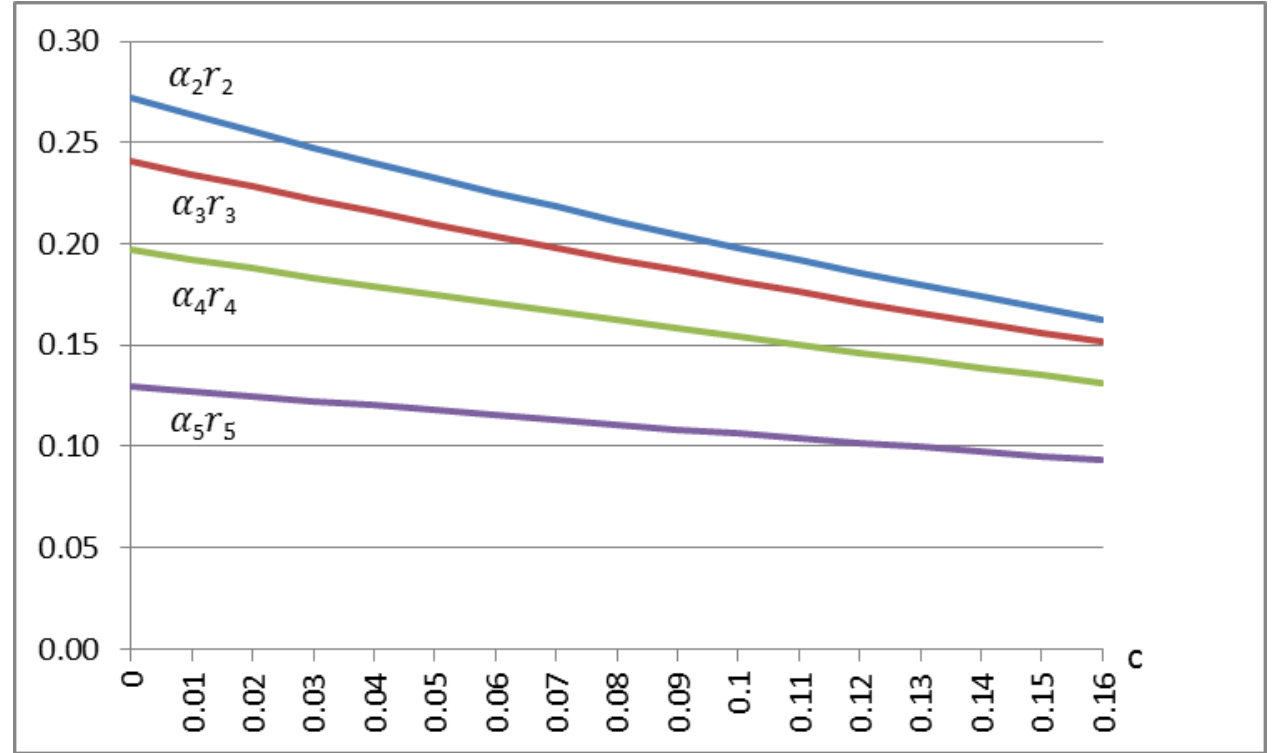

Notes: $\mathrm{n}=5$ and $\rho=0.01$. 